\title{
ANTROPOLOGÍA
}

\section{ANTROPOLOGÍA EDUCATIVA DE LOS CUIDADOS ENFERMEROS ANTROPOLOGÍA EN URGENCIAS}

\author{
César RICO BELTRÁN* \\ Elena ANDINA DÍAZ* \\ Pilar BONET MANSO* \\ COLABORADORA: SUSana CANET BELTRÁN \\ *Enfermeros Asistenciales. Hospital de Elda. Alicante.
}

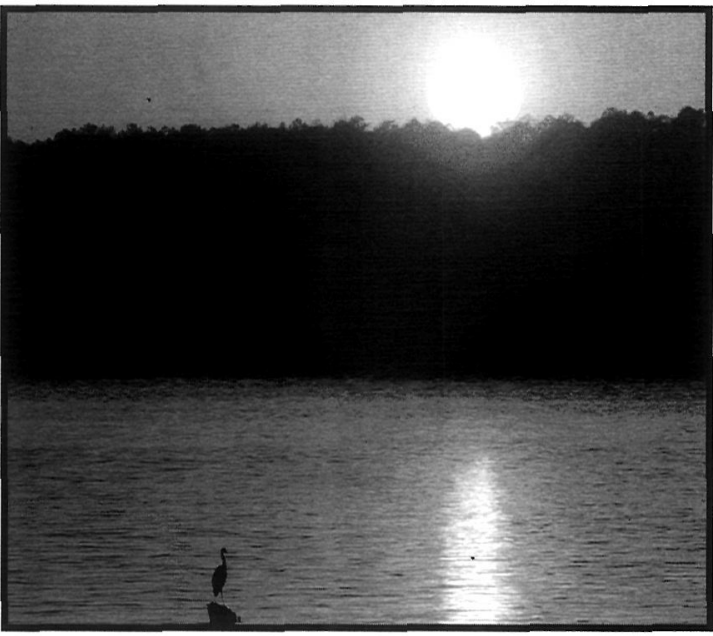

\section{ANTHROPOLOGY IN ACCIDENT AND EMERGENCY DEPARTMENTS}

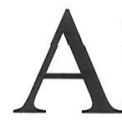

ccident and emergency departments become a meeting point for people with different concepts of health and illness. Using the phenomenologic theory, we have tried to describe and interpret the realm and ways in which these people interact.

In this work we focus on the study of the culture transmission / acquisition processes between the patients and health professionals, and on the description of the scene and atmosphere in which these processes occur.

The chosen method is participating observation, watching patients in the waiting room and cubicles, and wearing a uniform accompanying professionals in their duty, using the "key person" technique to be able to enter the scene of the investigation.

The study was carried out during April, May and June 2001 at the Accident and Emergency Department in Elda Hospital, Alicante.

\section{RESUMEN}

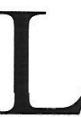

as puertas de urgencias de los hospitales son un punto de encuentro de poblaciones con distinto concepto de los términos salud y enfermedad. Fundamentándonos en la teoría fenomenológica hemos pretendido describir e interpretar el marco y las formas en las que se realiza la interacción de estas poblaciones.

En el presente trabajo nos hemos centrado en el estudio de los procesos de transmisión/adquisición entre la cultura de los pacientes y de los profesionales sanitarios y en describir el ambiente y escenario en el que se producen dichos procesos.

El método que utilizamos fue la observación participante realizando simultáneamente dos observaciones, una a los pacientes, introduciéndonos como meros observadores en la sala de espera y boxes del servicio y otra poniéndonos un pijama y acompañando a los profesionales en su trabajo, utilizando la técnica de personas clave para poder entrar en el entorno de la investigación.

El estudio se realizó durante los meses de abril, mayo y junio de 2001 en la puerta de Urgencias del Hospital de Elda (Alicante). 


\section{INTRODUCCIÓN JUSTIFICACIÓN}

Las "Áreas de Urgencias Hospitalarias" son la puerta de entrada al entorno sanitario más utilizada por algunos sectores de la población general; por lo que desde un ámbito antropológico, describir el intercambio cultural que en ese área se produce nos pareció un objetivo de estudio interesante.

Desde el punto de vista de la fenomenología, la descripción de estos actos refieren una conciencia propia de sí mismo y encierran una intención y una peculiaridad propia que debe darse en estos servicios y no en otros (San Martín, 1994).

La fenomenología es un análisis trascendental que supera a los sujetos, tratando al ser humano, no sólo su exterioridad, sino como ser del mundo, y éste es un aspecto que hemos querido resaltar en nuestro estudio (Husserl, 1982).

La utilización de estudios de investigación cualitativa está en auge actualmente en el área de la salud, como lo demuestra una revisión de estudios cualitativos en este campo realizada en los últimos años (De la Cuesta, 1994), en los cuales se muestra que han contribuido a describir y comprender el uso de los servicios de salud, describir y analizar las relaciones de los profesionales de la salud con los usuarios y las relaciones entre los profesionales, identificar y describir las diferentes "culturas" de los usuarios y profesionales de la salud.

Los cuatro métodos de investigación cualitativa son: "etnoenfermero", "etnográfico", "teoría fundamentada" y "fenomenología". Nos pareció interesante utilizar este último porque la fenomenología busca "comprender cómo las personas perciben su mundo y le dan un significado, permite que el investigador no solamente describa sino que también interprete las experiencias vividas por las personas" (Zoucha, 1999).

Para realizar la búsqueda de información de los trabajos realizados anteriormente sobre el tema de los fenómenos culturales en la sala de urgencias entramos en las bases de datos de Medline, Cinalh, Phyclit y Cuiden, utilizando las palabras clave: "cuidados de enfermería", "antropología de la salud", "cultura, antropología y urgencias", "enfermería y urgencias", "antropología y enfermería", "antrophology", "emergency", "emergency and nursing", "antrophology and nursing", no encon- trando ningún artículo que describiera cualquier estudio similar al que nosotros pretendíamos realizar, por lo cual nos pareció de interés, al tiempo que innovador, acometer este estudio.

\section{HIPÓTESIS DEL TRABAJO}

En el Servicio de Urgencias se produce un choque cultural entre el grupo de pacientes y el personal sanitario.

\section{OBJETIVOS}

- Describir el ambiente/escenario en el que se produce el choque intercultural paciente/profesional.

- Explicar los procesos de transmisión/adquisición de información entre cultura de pacientes y profesionales sanitarios.

\section{MATERIAL Y MÉTODO}

Para la realización del trabajo hemos empleado la "metodología cualitativa" que nos "permite constatar la existencia de una concepción simbólico-cultural sobre la salud, más amplia que los discursos médicos dominantes" (Leininger, 1991). Este tipo de metodología es útil cuando existe una gran carga de significados, estereotipos y perjuicios asociados a un tema, como sucede en nuestro caso, las urgencias.

Dentro de los "métodos cualitativos" existentes, nos pareció más apropiado "la observación participante", que nos permitía seguir, sin implicarnos al ser meros observadores, "los discursos tanto de las personas que trabajan en el sistema sanitario, como el de las personas a las que van dirigidas las prestaciones; permitiendo conocer también las interacciones entre ambos" (Amezcua, 2000). Posteriormente realizamos un análisis e interpretación del lenguaje, y finalmente extrajimos las conclusiones pertinentes (De la Cuesta, 1997).

Tomamos la información oportuna durante varias sesiones realizadas en los meses de Abril, Mayo y Junio de 2001 hasta asegurarnos de que el número de observaciones era suficiente, ya que ésta comenzaba a "ser reduntante, los datos eran repetitivos y no generaban conceptos ni teorías nuevas" (Amezcua, 2000); así podíamos "sacar conclusiones acerca de formas típicas o atípicas de 
comportamiento" (Miralles, Bangro, 1997) y, por lo tanto, habíamos llegado al punto de saturación. Al final del estudio se realizó la triangulación de los datos, reuniéndose el equipo para comparar los resultados y controlar las tendencias de los observadores y comprobar si "todos estaban llegando al mismo punto de saturación" (Amescua, 2000).

Para introducirnos en el campo de trabajo contactamos previamente con la Adjunta de Enfermería del Área de Urgencias, la Adjunta de Formación y Docencia y la Supervisora del Servicio de Urgencias del Hospital de Elda, que nos otorgaron el oportuno consentimiento oral y escrito.

Las pequeñas y únicas dificultades se encontraron a la hora de la ejecución, en cómo establecer contacto con los usuarios y/o pacientes, para que éstos contaran sus experiencias; sin embargo todas se solventaron al momento.

\section{RESULTADOS Y DISCUSIÓN ENTORNO FÍSICO}

El estudio lo realizamos en el Hospital de Elda. Este centro es el Hospital Comarcal del Área 17 de la provincia de Alicante, con una población de 191.320 habitantes (según Padrones Municipales, Instituto Nacional de Estadística 1/1/1998). Dispone de 274 camas, lo que significa que tiene 1,43 camas por cada 1.000 habitantes.

Las actividades económicas principales en la comarca son: la industria del calzado, explotación del mármol y el cultivo de la vid. En ese contexto económico es destacable la frecuencia con la que, al hablar con la población, muchos reconocen estar inmersos o realizar actividades económicas "sumergidas", así como la normalidad con la que aceptan esta situación.

El Hospital está situado dentro del término municipal de Elda. Su ubicación resulta estratégica por tres motivos:

1.- Es una población situada céntricamente dentro del área geográfica de referencia. (Ver anexo 1).

2.- El núcleo urbano formado por las poblaciones de Elda y Petrer concentran más del $40 \%$ de la población total. (Ver anexo 2)

3.- Política y administrativamente Elda es centro de la comarca.

Con respecto a la población de Elda, el
Hospital está situado fuera del núcleo urbano, próximo a la autovía, lo que facilita la comunicación con las principales poblaciones de su área y también la comunicación con el Hospital del siguiente nivel (Alicante).

El Servicio de Urgencias del Hospital de Elda, atendió en 1999, un total de 54.170 urgencias (ver anexo 3). En él las puertas de Urgencias son únicas, es decir, no hay unas puertas de Urgencias de materno infantil y otras generales. Sólo hay una puerta de Urgencias general donde se atiende todo (salvo la ginecología y obstetricia que se deriva a partos). Desde un punto de vista material, se trata de unas puertas de Urgencias pequeñas en las que no hay diferenciación por niveles de atención, ni equipo de triage, de manera que las personas que acuden para ser atendidas se concentran en una sala de espera común de adultos, y otra de pediatría. Los pacientes que están encamados ocupan primero los boxes de camas (boxes del 3 al 6); luego los huecos de observación de Urgencias, si son adultos (10 camas para adultos); o las camas del servicio de Pediatría, si son menores de 14 años. Después se ocupa la antesala del quirófano de Urgencias y finalmente los pasillos, dándose, con relativa frecuencia, el caso en que el número de pacientes excede la capacidad física de la Unidad, acomodándoles en un "hall" interior que comunica con el resto del Hospital. (Ver anexo 4).

(Fuente: Memoria 1999 Hospital Comarcal de Elda).

\section{OBSERVACIÓN PARTICIPANTE CON LOS PACIENTES}

\section{"SALA DE ESPERA"}

Nos sentamos en una silla de la Sala de Espera.

Los pacientes no se diferencian de los familiares o acompañantes. Sólo algunos, ya que van en silla de ruedas, y otros (pocos) que visten el "uniforme" del Hospital: el camisón. Todos se encuentran sentados, acompañados generalmente de una persona. Hay gente de todas las edades: los jóvenes suelen estar acompañados por mujeres de mediana edad, sus madres, o amigos; la gente de mediana edad, por su pareja (deducimos) al ser del sexo contrario; los ancianos vienen acompañados de mujeres más jóvenes que ellos. 
Observamos, sobre todo, que abunda la gente de nivel socioeconómico bajo y gitanos. Lo deducimos por sus vestimentas y rasgos físicos. También hay gente de clase media, bien vestida, predominando la de mediana edad.

No hacen barullo. Apenas se les oye hablar entre ellos, haciéndolo sobre temas triviales. Se observan caras serias, de aburrimiento o resignación.

$\mathrm{Al}$ fondo hay un mostrador. Varias personas, apoyadas en él, miran hacia dentro, como buscando a alguien, pero ese alguien (profesional) no aparece en escena.

Hay muchas puertas: "box 1", "box 2",.. De ellas entran o salen sanitarios, que aparecen con una hoja en la mano y gritan un nombre. El usuario, que se hallaba sentado en la sala, se levanta y le sigue. Sin haberse dirigido la palabra, ni siquiera para saludar, ambos entran en un "box". Al rato se descorre la cortina. Sale el profesional, rápido, y detrás el paciente.

Los celadores acuden al lugar donde los pacientes están en silla de ruedas, y sin mediar palabra se los llevan de un sitio para otro. En otras ocasiones se paran a hablar con ellos o les saludan.

Dos sanitarios intercambian palabras en medio de la sala. Ambos con hojas en la mano, uno le recrimina algo al otro, señalando los papeles.

- Pregunto a una mujer si lleva mucho tiempo esperando. Me responde que ha llegado, le han tomado la tensión y le han puesto esas pegatinas ("electro"), y que como supone que no le habrán encontrado nada malo, le han mandado que se siente en la sala y espere. No le han dicho cuánto tiempo esperará ni qué es lo que tiene. Se levanta para llamar por teléfono a su hijo.

- Una chica se queja de que le duele la muñeca. Ayer por la noche se cayó de la cama, y ha decidido ir hoy a las doce del medio día a Urgencias. Después de ser atendida nos comenta: "parece mentira, es para denunciarlos", y es que le han dicho que no tiene nada roto, "sin haberme hecho ni siquiera una radiografía me mandan a casa"...

- Otra chica cuenta que ha decidido venir a Urgencias porque sus padres le han obligado, porque ella se encuentra bien.

- Dos mujeres dialogan sobre lo que les dirá el médico: "seguro que me mira por encima y me mandan volver dentro de dos meses"; la otra añade: "no creo, a mí la última vez...".

\section{"EN BOXES"}

En "boxes" la situación es un tanto caótica debido a una imprevista aglomeración de pacientes. Camillas en los "boxes", en los pasillos, por el control de enfermería. No paran de traer enfermos. Los pacientes vienen vestidos (algunos a medias) con pijama del Hospital, puestos en la camilla o en la silla de ruedas, aglomerados en medio del pasi1lo. Los familiares al lado de los enfermos, de pie, dándoles la mano y hablando con ellos.

Los pacientes están, generalmente, desorientados. Preguntan a los profesionales sobre todo: “¿qué me pasa?, ¿cuánto tiempo estaré aquí?’. Los familiares preguntan “¿quién me puede informar?, ¿puedo estar aquí?, ¿por dónde salgo a la calle?’. Una chica saca su móvil de la bolsa que tiene, con sus ropas, encima de su cama, y escribe un mensaje. Le comenta a su madre: "le he dicho que estoy bien".

El pasillo es vía de paso, no sólo del personal sanitario y pacientes; también de gente de la calle, personal de mantenimiento como fontaneros, electricistas...

No hay contacto físico entre pacientes y profesionales. Los médicos permanecen cruzados de brazos mientras dialogan con los pacientes y familiares. Sólo vemos contacto físico cuando hay que realizar alguna técnica invasiva. Vemos a una enfermera que le extrae sangre a un señor que está en una camilla, en medio del pasillo.

Los profesionales van y vienen a su aire. Les vemos, como locos, buscando "boxes" o camillas impacientemente, sin prestar atención a goteros que se acaban o a familiares que les llaman. Algunos profesionales llevan en su uniforme escrito su cargo "Médico", "D.U.E.", pero la mayoría no llevan nada.

\section{OBSERVACIÓN PARTICIPANTE CON PROFE- SIONALES:}

\section{PROCESO DE ATENCIÓN}

Para esta observación nos pusimos un pijama y una bata y acompañamos a los profesionales durante varios turnos de trabajo.

A primera hora, los enfermeros salientes cuentan a los entrantes las novedades del relevo: 
los pacientes que tienen vistos y las tareas que quedan por realizar (cambiar un gotero, canalizar vía...). Las auxiliares de enfermería, los celadores y administrativos no "se cuentan" el relevo, los médicos informan a los entrantes sobre los pacientes que tienen vistos. No se hace el relevo de los médicos simultáneamente al del resto de profesionales.

Tras el relevo, se realizan las tareas pendientes y revisan los "boxes" de atención. Reunión para tomar el café, en que se habla de temas triviales: familiares, de prensa rosa, deportivos...

Los primeros en "ver" a los pacientes son los enfermeros que comienzan con la valoración y la toma de constantes. Después los visita el médico que apunta las pruebas que se le han de realizar y la medicación que hay que ponerle; no suelen informar al paciente ni de las pruebas a practicar, ni de las sospechas diagnósticas. Curiosamente el personal sanitario comenta a la mayoría de los pacientes que no tenían que haber acudido al Servicio de Urgencias hospitalarias, por no existir una causa urgente y grave; pero allí se les practica una amplia serie de pruebas especializadas (analíticas, radiografías, etc.) en un período relativamente corto de tiempo, como los pacientes desean; todo parece ser una contradicción.

Los enfermeros canalizan vías, extraen sangre, administran medicación, realizan vendajes... y cursan pruebas diagnósticas y hojas de interconsulta.

Los médicos dan el alta al paciente de modo verbal primero, y luego por escrito; explicándole el diagnóstico, la pauta del tratamiento y los cuidados que debe seguir.

\section{INTERACCIONES}

Los profesionales no suelen identificarse por su nombre; si bien, en ocasiones, se identifican por su categoría (los médicos lo hacen siempre por su categoría).

Las quejas las reciben normalmente cualquier profesional menos el médico. Los pacientes suelen comportarse de forma más respetuosa con los facultativos que con el resto de personal, aunque algunos tengan con los pacientes una actitud fría y distante. Las quejas más frecuentes suelen referirse al tiempo de espera, al número de acompañantes.
Los pacientes demandan información al médico sobre su diagnóstico; y sobre el proceso de atención restante a los enfermeros: qué pruebas les van a realizar, cuánto tiempo van a estar allí,...

\section{- RESULTADOS DE LA OBSERVACIÓN A PACIENTES: \\ ¿CÓMO VAN VESTIDOS?}

Las mujeres y hombres de edades comprendidas entre 40 y 70 años suelen acudir al Servicio de Urgencias arreglados, vestidos de una manera un tanto formal, mientras que los más jóvenes llevan ropas más cómodas tipo chándal, vaqueros, zapatillas de deporte, etc.; tal vez esto se deba a un cambio de actitud generacional a la hora de concebir el significado "ir al médico", al papel de menor protagonismo que a éste (como a otros muchos profesionales) se les da actualmente en la sociedad. Por su vestuario se deduce que pertenecen a un nivel económico medio-bajo, y por su conversación y expresiones, en el habla, a un nivel instructivo bajo.

\section{¿CUÁLES SON LOS DISCURSOS CON CADA PROFESIONAL?}

La comunicación paciente-profesional sanitario apenas existe, al menos fuera de la consulta. Los pacientes cuando son llamados siguen al profesional que, sin mediar palabra y con gran rapidez, se dirige a un "box".

En el nivel 1 no existe contacto con el paciente salvo para realizar técnicas invasivas. Aunque no haya intercambio de palabras entre profesional sanitario y paciente, sí existe un lenguaje corporal (como mantener los brazos cruzados) que advierte de que debe ser así (manteniendo las distancias).

Las preguntas más frecuentes que los pacientes realizan a los profesionales de la salud son: ¿qué me pasa?, ¿cuánto tiempo estaré aquí?; mientras que las de sus familiares son: ¿por dónde salgo a la calle?, ¿puedo estar aquí?, ¿quién me hace caso?

\section{NÚMERO DE ACOMPAÑANTES Y RELA- CIÓN PARENTAL}

La mayoría suelen ir acompañados de sus parejas a excepción de las chicas jóvenes que van acompañadas de sus madres, y en algún caso de su madre y una amiga. 


\section{¿DE QUÉ HABLAN ENTRE ELLOS?}

Tanto los pacientes como sus familiares, con aspecto relajado, hablan de temas triviales sin importancia y sin mencionar el asunto por el que acuden a Urgencias. En algunos casos comentan con el que se encuentra más próximo el diagnóstico que ellos mismos hacen de su problema.

\section{COMPORTAMIENTOS}

Los pacientes permanecen sentados esperando a ser atendidos, mientras que sus familiares entran y salen, y además hacen alguna que otra llamada desde su móvil.

\section{¿POR QUÉ HAN ACUDIDO A URGENCIAS?}

La decisión de acudir al servicio de Urgencias no proviene del mismo afectado, mas bien ésta se decide en el ambiente familiar, en el lugar de trabajo o incluso entre los vecinos, que después de hacer su propio diagnóstico, aconsejan y en muchos casos imponen que se acuda al Servicio de Urgencias del Hospital.

\section{- RESULTADOS DE LA OBSERVACIÓN A PROFESIONALES:}

\section{¿VAN IDENTIFICADOS?}

La normativa oficial del centro dice que todos los profesionales deben de llevar una tarjeta de identificación que indique el nombre, apellidos, categoría profesional y unidad en la que trabaja; además, para facilitar la identificación de la categoría profesional, estas tarjetas son de color distinto en función de dicha categoría.

Hemos observado que aunque los profesionales reconocen que las tarjetas de identificación son un buen medio de comunicación pasiva, la utilización por su parte es desigual: los hay que la llevan siempre, pero también existen otros que se sienten incómodos llevándola. Al preguntarles los motivos por los cuales no la llevaban, las razones iniciales que daban es que resultaba incómoda porque se enganchaba en todos los sitios, que la habían olvidado en la taquilla o que se les había roto la funda que la sujeta. Respecto a los profesionales con los que hemos podido entablar mayor comunicación, han dado los siguientes argumentos para no llevarla: "si doy datos acerca de mi identidad es más probable que me puedan denunciar", "me da vergüenza llevar un cartel con mi nombre y mi cara" y "ahora les da porque nos pongamos esto, luego les dará por otra cosa".

Analizando si existía una identificación activa por parte de los profesionales, estuvimos observando si se presentaban a los pacientes indicando su categoría profesional, y pudimos constatar que los profesionales que más frecuentemente comunicaban su categoría profesional al paciente eran los médicos, seguidos, con bastante diferencia, por los enfermeros. Resultaba muy raro que las auxiliares de enfermería y los celadores se identificaran.

\section{¿QUÉ PREGUNTAN Y QUÉ DICEN CADA UNO DE LOS PROFESIONALES?}

Administrativos. Preguntan los datos del paciente y el motivo por el que vienen. En caso de accidentes los datos de la mutua. Las preguntas a las que responden se refieren a los familiares y acompañantes que están en la sala de espera, y lo que éstos quieren saber sobre si su familiar está ya atendido o ingresado en la Unidad.

Celadores. Preguntan sobre el motivo por el que vienen a Urgencias y si pueden caminar por sí solos. No suelen responder a las preguntas, cuando se les interroga sobre algo derivan a otros profesionales.

Auxiliares de enfermería. En la parte de pediatría preguntan la edad del paciente y el motivo por el que vienen; en la parte de adultos preguntan acerca de la movilidad del paciente, del control de esfinteres y por la presencia o no de familiares. Suelen derivar las preguntas que se les hacen hacia otros profesionales.

Enfermeras. Preguntan sobre el motivo por el que han venido a Urgencias, los síntomas que presentan y la evolución del proceso, sobre las alergias medicamentosas, si presentan otras enfermedades de interés como la diabetes, si trae acompañantes o no, y por el grado de independencia del paciente. En la zona de pediatría preguntan además por la edad del paciente. Contestan a preguntas referidas al tratamiento, la posible evolución del proceso, el nivel de gravedad del paciente, el especialista que lleva al paciente, y sobre los cuidados que deben seguirse, tanto en el período hospitalario, como tras el alta.

Médicos. Preguntan sobre el motivo por el que han venido los pacientes a Urgencias, sobre su his- 
toria clínica (alergias, enfermedades previas, intervenciones quirúrgicas, ingresos hospitalarios, etc.). Responden a preguntas relativas al diagnóstico y tratamiento de la enfermedad, sobre la posible evolución del proceso, de las pruebas complementarias que deben de realizársele al paciente y de los especialistas que lo deben ver.

\section{¿QUIÉN RECIBE Y QUIÉN SE QUEDA CON EL PACIENTE?}

La recepción del paciente la realiza el celador o la administrativa que son las personas que están en la puerta de entrada. Si consideran que el paciente está grave lo presentan a una enfermera para que lo valore y decida si hay que atenderlo en ese momento, o si por el contrario se puede demorar la atención, caso de que haya otros pacientes esperando. Tras esta valoración, si el paciente está en una situación grave o crítica se comienza a atenderle, de inmediato, siendo la enfermera quien pauta las normas de actuación, mientras se avisa a un médico para que acuda; una vez visto y valorado por éste, el paciente queda bajo los cuidados de la enfermera y la auxiliar de enfermería. Si tras la valoración de la enfermera, la atención del paciente puede ser demorada, éste permanecerá en la sala de espera hasta que le vea el facultativo correspondiente; si después de esta vista el paciente necesita de alguna atención, ésta será realizada por las enfermeras o por las auxiliares de enfermería.

\section{¿QUIÉN LIDERA EL GRUPO Y ORGANIZA EL TRABAJO?}

La figura del médico es la que tiene mayor liderazgo en cuanto a la atención del paciente, ejerciéndolo mediante órdenes verbales en el momento de la atención y a través de la prescripción de tratamientos. No obstante la enfermera adquiere un gran protagonismo en la clasificación de los pacientes, a la hora de decidir el orden en que deben ser atendidos y la ubicación que debe tener un paciente. En definitiva, se podría decir que en la atención de un paciente el médico lidera el grupo, mientras que en la planificación de la unidad y en el control de las situaciones de crisis (múltiples victimas, colapso por falta de espacio, etc.) son las enfermeras las que organizan el trabajo y el Servicio de Urgencias. No obstante el liderazgo en un servicio de Urgencias y en la atención a un paciente se rige por las mismas normas que las de liderazgo de cualquier grupo humano, y no es extraño ver cómo enfermeras con especial habilidad y reconocido prestigio lideran y dirigen la atención y cuidado de un paciente, y organizan el servicio. Sin embargo, no hemos constatado que en ningún momento el celador, el administrativo o la auxiliar de enfermería liderasen el grupo.

\section{CONCLUSIONES, UTILIDAD Y LIMITA- CIONES}

Podemos concluir diciendo que las puertas de urgencias hospitalarias son un lugar de choque o contraste cultural entre dos percepciones distintas del proceso salud-enfermedad; por una parte, la de los pacientes, que en su percepción utilizan un paradigma sociocultural, la enfermedad subjetivamente experimentada (illnes); y, por otra, la de los profesionales, que utilizan un paradigma biosanitario, la enfermedad científicamente observada (disease). Como resultado de este choque hemos observado que el estamento de mayor poder, en este caso el sanitario, es el que impone su paradigma, siendo los pacientes los que cuando hablan con ellos adaptan su lenguaje al lenguaje sanitario (Bernalte, et al 1999).

También existe una confrontación en cuanto a las expectativas de ambos grupos; de una parte, los pacientes que piensan que al acudir al hospital acceden al nivel máximo de conocimientos sanitarios y en las puertas de Urgencias dispondrán de ese conocimiento de manera inmediata (se realizan pruebas de forma rápida) y pudiendo elegir ellos el momento en que acceden a esa atención. Por otra parte, los profesionales sanitarios viven esa situación de manera distinta, percibiendo la escasez de espacio, tiempo y recursos, incluso de solicitud de pruebas, dándose cuenta de las limitaciones del servicio que ellos ofrecen. (Husserl, 1992)

Por lo tanto, se confirma la hipótesis de que en el Servicio de Urgencias se produce un choque cultural entre el grupo de profesionales sanitarios y el grupo de pacientes. 


\section{BIBLIOGRAFÍA}

AMEZCUA, M. (2000) El trabajo de campo etnográfico en salud. Index de Enfermería, Año XI, n 3. Otoño

BERNALTE BENAZET, A; ET AL (1999). Atención a los cambios culturales y a su impacto en la salud: Memoria de un pequeño trabajo de campo. Enfermería Científica, n 202-203, Ene-Feb.

DE LA CUESTA, C. (1997) Investigación cualitativa en el campo de la salud. Revista Rol de Enfermería, $n^{\circ} 232$

DÍAZ, C. (1998) Fenomenología de Hegel. Editorial Alambra. Madrid (1998).

FERNÁNDEZ DE SANMANUEL, M.J. (2000) Adecuación de las normas de publicación de revistas científicas a la investigación analítica. Atención Primaria, vol. 25, n 7, 30 Abril

HEIDEGGER, M. (1992) La fenomenología del espíritu de Hegel. Alianza Editorial. 1992.

HUSSERL, E. (1982) La idea de la fenomenología. Fondo de Cultura Económica. México, DF
HUSSERL, E. (1992) Invitación a la fenomenología. Ed. Paidos-Iberia. UCE/UAB.

LEININGER, M. (1991) Culture care Diversity and Universality: A Theori of Nursing. New York. National League for Nursing.

MIRALLES BANGRO, M.F.; ET AL. (1997) Elaboración de un trabajo práctico sobre la antropología de los cuidados. Cultura de los Cuidados, 1(2): 40-3

ROHKBACK, C. (1997) Cuidar es antiguo como el mundo y tan cultural como la diversidad humana. Cultura de los Cuidados, 1(2): 36-9

SPECTOR, R. (1999) Cultura, cuidados de salud y enfermería. Cultura de los Cuidados, 3(6): 66-79 Hospital General de Elda: Memorias 1999 Generalitat Valenciana.

ZOUCHA, R. (1999) La utilización de métodos cualitativos en enfermería. Cultura de los Cuidados, 3(6): $80-90$

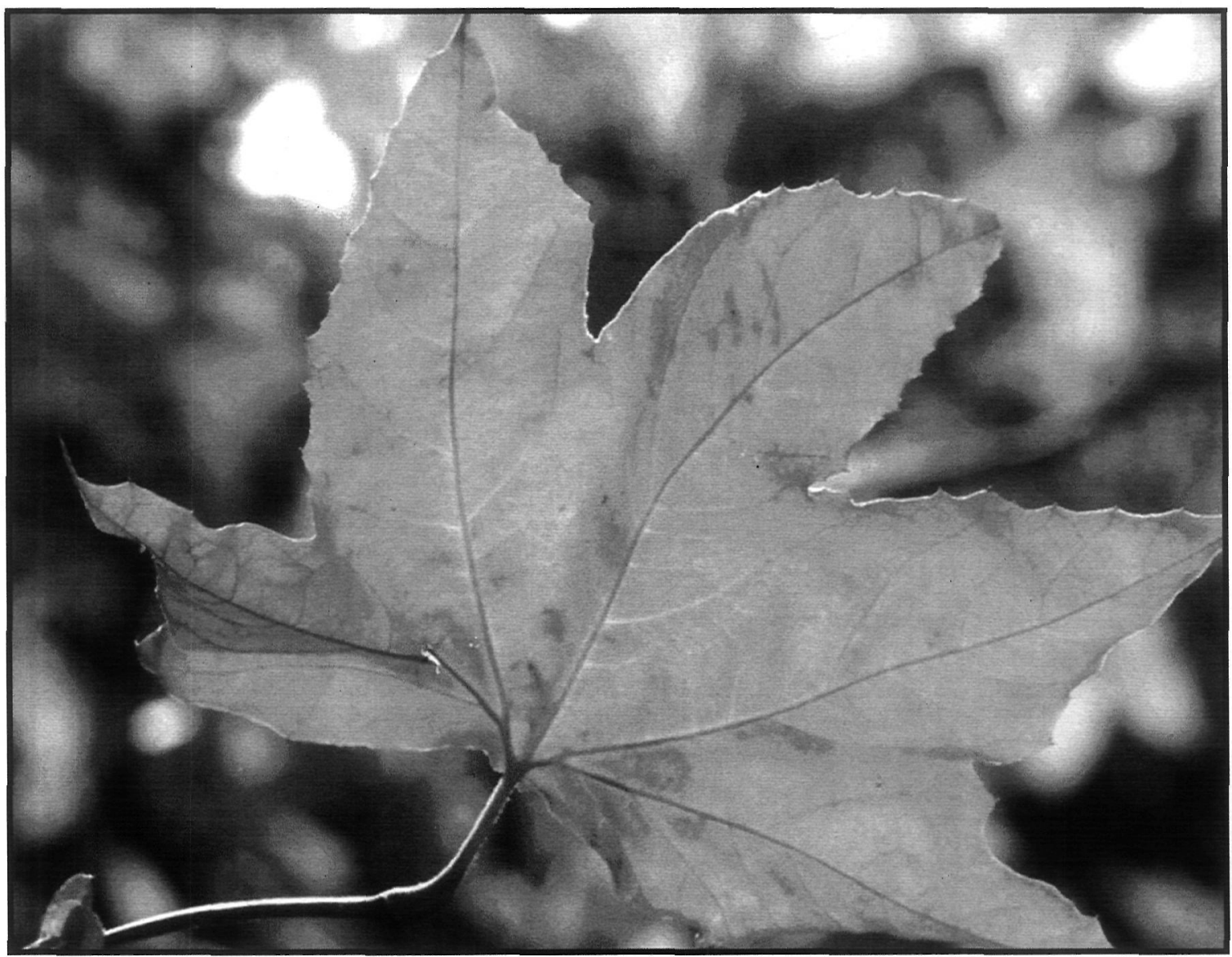




\section{ANEXO 1}

Área 17 (Frecuentación de urgencias por zonas)

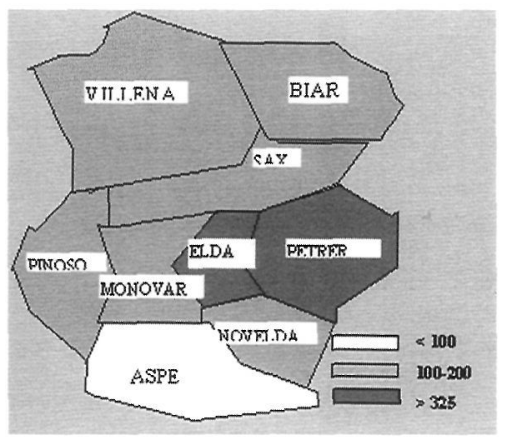

Fuente: Extraído de la memoria de 1999 del Hospital de Elda

\section{ANEXO 2}

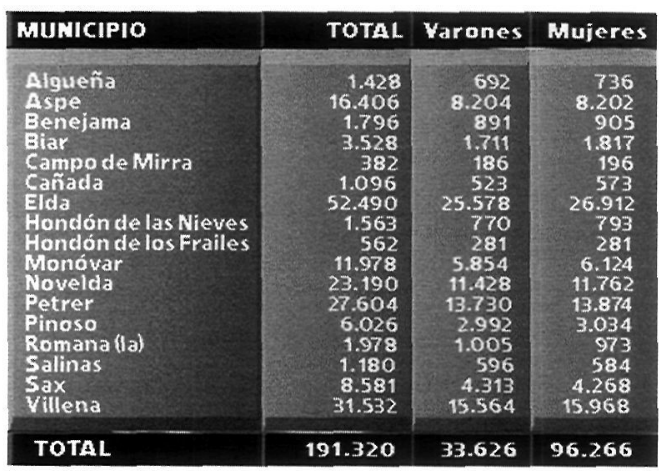

Fuente: Extraído de la memoria de 1999 del Hospital de Elda

\begin{tabular}{|c|c|c|c|c|c|c|c|c|c|c|c|c|c|}
\hline Población & Ene. & Feb. & Mar. & br. & Mav & un. & al. & Ago. & Sep. & Oct. & Nov. & Dic. & Total \\
\hline Algueña & 22 & 20 & 20 & 28 & 20 & 24 & 17 & 27 & 30 & 31 & 16 & 30 & 285 \\
\hline Aspe & 14 & 13 & 10 & 12 & 14 & 15 & 13 & 8 & 13 & 15 & 12 & 9 & 148 \\
\hline Benejama & 35 & 48 & 48 & 29 & 38 & 23 & 27 & 22 & 24 & 45 & 30 & 23 & 392 \\
\hline Biar & 57 & 39 & 42 & 52 & 60 & 33 & 52 & 62 & 57 & 47 & 40 & 55 & 596 \\
\hline Campo de Mirra & 7 & 4 & 2 & 4 & 3 & 2 & 6 & 4 & 4 & 5 & 6 & 4 & 51 \\
\hline Cañada & 9 & 12 & 17 & 8 & 17 & 14 & 13 & 15 & 20 & 24 & 20 & 17 & 186 \\
\hline Elda & 1.651 & 1.698 & 1.705 & 1.565 & 1.691 & 1.697 & 1.671 & 1.700 & 1.664 & 1.724 & 1.543 & 1.581 & 19.890 \\
\hline Hondón Nieves & 0 & 2 & 0 & 2 & 0 & 0 & 2 & 1 & 1 & 1 & 4 & 5 & 18 \\
\hline Hondón Frailes & 0 & 0 & 1 & 0 & 0 & 0 & 0 & 2 & 1 & 0 & 1 & 2 & 7 \\
\hline Monóvar & 337 & 342 & 327 & 274 & 327 & 311 & 312 & 374 & 338 & 354 & 321 & 292 & 3.909 \\
\hline Novelda & 582 & 576 & 558 & 488 & 523 & 504 & 516 & 612 & 601 & 556 & 506 & 628 & 6.650 \\
\hline Petrer & 821 & 721 & 756 & 756 & 816 & 732 & 801 & 716 & 718 & 832 & 755 & 769 & 8.193 \\
\hline Pinoso & 109 & 91 & 115 & 97 & 81 & 104 & 107 & 96 & 94 & 97 & 97 & 116 & 1.204 \\
\hline Romana, La & 30 & 33 & 44 & 26 & 24 & 29 & 35 & 51 & 31 & 28 & 29 & 40 & 400 \\
\hline Salinas & 29 & 49 & 23 & 31 & 26 & 21 & 35 & 27 & 42 & 27 & 31 & 19 & 360 \\
\hline Sax & 250 & 252 & 206 & 216 & 236 & 231 & 220 & 230 & 241 & 222 & 219 & 199 & 2.722 \\
\hline villena & 540 & 519 & 552 & 500 & 526 & 553 & 539 & 616 & 511 & 562 & 512 & 519 & 6.449 \\
\hline Otros & 125 & 115 & 133 & 150 & 144 & 122 & 146 & 188 & 121 & 189 & 159 & 118 & 1.710 \\
\hline TOTALES & 1618 & 4534 & 4559 & 4238 & 4.546 & 4.415 & 4.512 & 4.751 & 4.511 & 4.759 & 4.301 & 4.426 & 54.170 \\
\hline
\end{tabular}

\section{ANEXO 4}

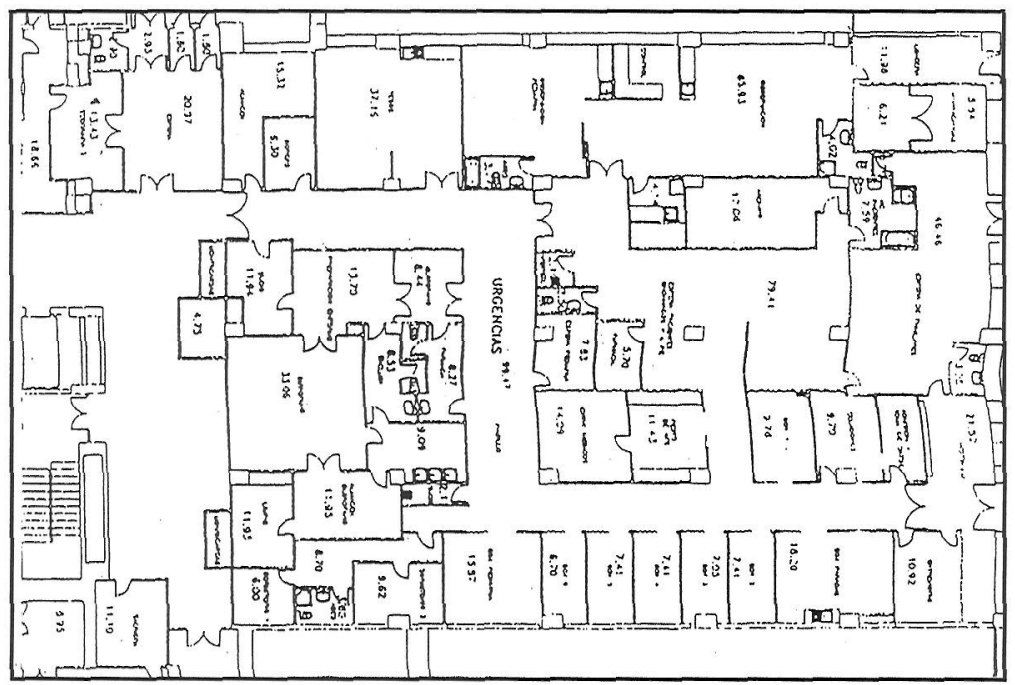

\section{ANEXO 3}

Urgencias mensuales (1999)

Fuente: Memoria de 1999 del Hospital de Elda 